\title{
Ultrasound Imaging of Brain with Machine Learning
}

\author{
A proposed machine-learning technique can convert ultrasound signals \\ into a skull profile, which could lead to noninvasive imaging for medical \\ treatments in the human brain.
}

By Rachel Berkowitz

1 arl Dussik was one of the pioneers of medical ultrasound imaging, using the technology to image human brains in the 1940s. However, the brain has remained difficult to measure accurately, as the skull distorts the acoustical signal, resulting in blurred images. Techniques exist for mitigating the effect of the skull, but they can be time consuming and can expose patients to invasive radiation. Researchers now demonstrate a machine-learning-based method that estimates the skull's thickness using ultrasound echo signals reflected from the skull. The resulting profile can then be used to correct

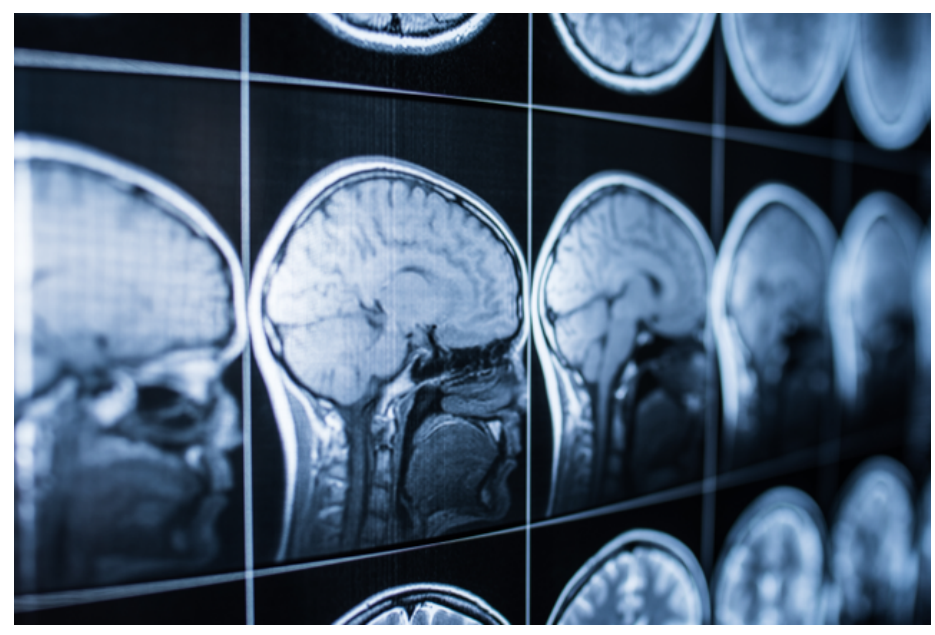

These brain images require $x$ rays, but a new ultrasound technique might one day provide similar results without harmful radiation. Researchers demonstrated a machine-learning method that helps overcome distortions caused by ultrasound signals traversing the skull.

Credit: jalisko/stock.adobe.com signal distortions, leading to the development of high-resolution, noninvasive neural imaging tools.

Many common medical ultrasound imaging applications directly target soft tissues, where the speed of sound is well known and relatively homogenous. Unlike soft tissue imaging, placing a skull in the acoustical path substantially modifies the propagation and acts as a wavy lens that distorts the beam. If you know the thickness of the lens at different locations, you can correct for that distortion. Thus, high-resolution ultrasound images of the brain require creating a profile of the skull that shows the thickness variations and speed of sound distribution.

The current best practice is to create individual skull profiles using computed tomography (CT) scans or magnetic resonance imaging (MRI). The profile provides exact knowledge of how the skull affects ultrasound propagation. Still, requiring an additional scan "defeats the ease of ultrasound," says Yun Jing from Pennsylvania State University. CT and MRI methods are resource and time intensive, and $\mathrm{CT}$ scans expose the brain to harmful radiation.

Jing and colleagues at Pennsylvania State University and at the University of Illinois at Urbana-Champaign (UIUC) propose a new method to extract the skull properties using radio-frequency (rf) ultrasound pulses reflecting off a skull. "It's really difficult to extract the skull's information from backscattered echoes because the underlying physical model is so complicated," says Jing. The researchers developed a machine-learning algorithm that can translate the echo data into a skull profile. The findings were presented at the end of November at the 181st meeting of the Acoustical Society of 
America, held in Seattle.

To test their method's feasibility, the researchers performed acoustic simulations using a realistic skull model built from CT scans of five human skulls. In their simulations, an ultrasound transducer transmits short ultrasonic waves that enter the skull and reflect back to the transducer, where they are recorded. The researchers fed the recorded echoes into a specially designed neural network that processes the signals and generates an estimate of skull thickness and speed of sound in the skull.

"Raw rf data contain a rich amount of information about the tissue," says UIUC team-leader Aiguo Han. Developing a neural network that can efficiently extract medical information from rf data is not trivial. Han's group had previously designed a neural network that worked well for accurately quantifying the percentage of fat in the liver, and that success "encouraged us to pursue a similar approach for the skull," he says.

The researchers trained their neural network by performing thousands of simulations of ultrasound signals echoing off the model skull. Comparing the network-estimated properties with measured data from the original CT scans yielded a mean error of $0.2 \mathrm{~mm}$ for the skull thickness and $25 \mathrm{~m} / \mathrm{s}$ for the sound speed prediction-representing model errors of $1-3 \%$. "Applying machine learning to the problem of predicting skull thickness and speed of sound is a very interesting and novel approach," says Greg Clement, a research physicist at the U.S. Food and Drug Administration's Office of Science and Engineering Laboratories, Maryland.

Successful implementation of this technique in humans could lead to vascular maps and active imaging of the brain with much higher resolution than, for example, MRI imaging, says Gianmarco Pinton, a biomedical engineer at the University of North Carolina at Chapel Hill. He adds, "it could also be performed in a more natural environment where the person can engage in activity, rather than being confined to a magnet." The result could provide a powerful tool for ultrasound brain imaging.

Rachel Berkowitz is a Corresponding Editor for Physics based in Vancouver, Canada. 